\title{
Non-local means image denoising with bilateral structure tensor
}

\author{
Li Huan ${ }^{1, a}, \mathrm{Xu} \mathrm{Yi}^{2, b}$ \\ ${ }^{1}$ Wuhan University of Technology, China \\ ${ }^{2}$ Wuhan University of Technology, China \\ aemail:1316531842@qq.com, bemail:365220180@qq.com
}

Keywords: Non-local means, bilateral structure tensor, image denoising, texture.

\begin{abstract}
Non-local means image denoising with bilateral structure tensor algorithm is put forward for the reason that Non-local means(NLM) algorithm has a weaker detail retention and noise immunity. Different from the initial structure tensor, we can get better texture description between similar blocks by using of bilateral structure tensor of noise resistance and texture features. New texture can improve the description of NLM value calculation function which filter the noise of images. Compared with traditional NLM algorithm, NLM-BST algorithm gets better image detail preservation in noise immunity. The experimental results show that operator is validated in denoising and image detail reservations.
\end{abstract}

\section{Introduction}

A good filtering algorithm can preserve the details of image texture while denoising, and Buades [1] and other scholars first proposed Non-local mean filtering (NLM) algorithm is to make full use of image self-similarity and image block to retain the image Texture detail features and complex spatial relations, so that it has a better denoising effect. However, the shortcomings of the algorithm in the following points: a certain noise intensity reduces the image block and block matching accuracy. weight calculation is taking into account a certain area including all similar compared blocks which increases the computation and time consumption of the algorithm.

In order to improve the accuracy of NLM algorithm in similar block matching, Liang Guangshun [2] and others used the spatial proximity in bilateral filtering and gray similarity to construct a new weight coefficient to improve the denoising effect. Yin Panpan et al. [3] used the modified Mahalanobis distance to analyze the image data and reduce the dimension. Finally, the improved Mahalanobis distance was used to generate the Gaussian weighted kernel function to denoise the image. The principal component analysis method proposed by Tasdizen improves the matching precision and reduces the matching. Deledalle C A [5] proposed an adaptive selection shape similarity measure and Yan [6] insert into NLM Framework by integrating Gaussian Blur, Clustering and Rotation Invariant Block Matching (RIBM) to improve denoising performance.

Based on the above mentioned improvements, the matching precision of similar blocks in image greatly determines the denoising effect to a great extend. Therefore, from the point of view of improving the similarity between image blocks, this paper introduces a double-structure tensor with certain robustness and anti-noise property [7], which can describe the texture between the image blocks, by rewriting the original NLM in the weight function to further improve the matching phase and improve the denoising while reducing the workload of the algorithm, from the perspective of optimizing the filtering performance.

\section{NLM algorithm principle}

For a given noise image, $\mathrm{v}=\{v(i) \mid i \in I\}$. The denoised image is defined as $N L[v](i)$. The pixel values at the $i$ point in the image are estimated by aver aging all the pixel values in a neighborhood with a similar neighborhood to the $i$ neighborhood, and the calculation is as follows: 


$$
N L[v](i)=\sum_{j \in I} w(i, j) v(j)
$$

In the formula (1), $I$ denotes the neighborhood centered on the pixel $j$, and the weight set $\{\mathrm{w}(\mathrm{i}, \mathrm{j})\}$

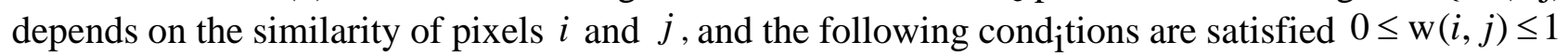
and $\sum_{\mathrm{j}} w(i, j)=1$, The weight function is defined as follows:

$$
\mathrm{w}(i, j)=\frac{1}{Z(i)} e^{-\frac{\left\|v\left(N_{i}\right)-v\left(N_{j}\right)\right\|_{2, a}^{2}}{h^{2}}}
$$

In the formula (2), $\mathrm{v}\left(N_{k}\right)$ is the gray intensity vector of all the pixels in the neighborhood. $N_{k}$ denotes the neighborhood of the pixel $k$ domain area. Similarity between blocks $\mathrm{i}_{\mathrm{S}}$ calculated by Gaussian weighted Euclidean distance, using $\left\|\mathrm{v}\left(N_{i}\right)-v\left(N_{j}\right)\right\|_{2, \mathrm{a}}^{2}$ to be measured. Where a is a Gaussian kernel with a deviation greater than 0 , the influence of noise on the distance calculation can be reduced, and the center of the pixel is highlighted. The smaller the gray intensity vector differences, the higher the similarity between the two, the greater the weight.

And $\mathrm{Z}(\mathrm{i})=\sum_{j} e^{-\frac{\left\|v\left(N_{i}\right)-v\left(N_{j}\right)\right\|_{2, a}^{2}}{h^{2}}}$ is the normalization constant and $\mathrm{h}$ is the attenuation function of the smoothing parameter control function.

\section{Research on NLM - BST Algorithm}

The structural information in the image is not only the basis of similar block comparison, but also the key to the quality of the algorithm. The traditional NLM algorithm uses the mean gray value to estimate the structure information of block and block in the image, but does not take into account the characteristics of the texture structure in the image, and it is also a factor that affects the similarity matching. And the structural tensor [8] is the extraction method of the spatial structure feature which conforms to the human visual characteristic. The feature similarity matching [9] of the spatial structure can obtain better denoising effect.

\section{Theoretical Analysis of Structural Tensor}

The theory of structural tensors was first proposed by W. Forstner [8] and applied to image feature detection. Defines the gray level $I$ of a two-dimensional image $I: \Omega \rightarrow R$, Where $\Omega \subset R^{2}$ is the data matrix of the two-dimensional image.The gradient vector of the two-dimensional data image is expressed as $\nabla I(i, j)=\left[I(i, j)_{x}, I(i, j)_{y}\right]^{T}$, The initial structure tensor $S_{0}$ and the two-dimensional structural tensor $S_{\omega}$ are expressed as follows:

$$
S_{0}(i, j)=\nabla I(i, j)(\nabla I(i, j))^{T}=\left[\begin{array}{cc}
I_{x}^{2}(i, j) & I_{x}(i, j) I_{y}(i, j) \\
I_{y}(i, j) I_{x}(i, j) & I_{y}^{2}(i, j)
\end{array}\right]=\left[\begin{array}{ll}
s_{11} & s_{12} \\
S_{21} & s_{22}
\end{array}\right]
$$

$$
S_{\omega}(i, j)=\sum_{x, y=-t}^{t} S_{0}(i-x, j-y)
$$

In the formula (3), $(i, j)$ represents the coordinates of the pixel points in the image $I . I_{x}(i, j)$ and $I_{y}(i, j)$ represent partial derivatives in the $x$ and $y$ directions, respectively. $\omega$ is a given $2 t+1$ 
square matrix.

The gradient vector calculated from different pixel points can be expressed as the edge structure feature and direction information at this point, but the gradient value at a single pixel point is the texture information of the image can not be traced. Using $S_{0}$ in (1) can get the image of rich local structure information, and can measure the anisotropy of structural features in the neighborhood of the pixel. The texture structure information is represented by the eigenvalues of the corresponding structural tensors, and the $S V D$ decomposition using the covariance matrix [8] yields the eigenvalues as follows:

$$
\mu_{1,2}=\frac{1}{2}\left(s_{11}+s_{22} \pm \sqrt{\left(s_{11}-s_{22}\right)^{2}+4 s_{12}^{2}}\right)
$$

The relation between $\mu_{1}$ and $\mu_{2}$ in the formula (5) reflects the local pixel texture structure, the specific relationship can be referred to [10] [11].

Structure tensor $S_{0}$ describes the superiority of image edge structure in low-intensity noise conditions, the partial derivatives of noise .The tensor can not describe the image structure correctly under certain intensity noise. In order to overcome the influence of noise on the tensor of structure, the Gauss convolution kernel $G_{\sigma}$ and the structural tensor $S_{0}$ are used for convolution. Although the image is smoothed to overcome some noise interference, the image edge information is weakened and blurred. The non-linear anisotropic tensor diffusion model given by Wieckert [12] et al. and the improved tensor model proposed by Perona and Malik [13] are based on suppressing noise intensity and preserving image edge details. However, considering the computational complexity of the non-local mean filter itself, it is difficult to reconstruct a new tensor model using the complex model described above. The complex model is used to reconstruct the new tensor model. Therefore, from the algorithm processing complexity and computational simplicity on the analysis, How to construct structural tensors with certain noise immunity and simple operation is the core of this paper.

\section{Anti - noise Model of Bilateral Structural Tensor}

Anti-noise interference and noise smoothing is the key to image detail processing. In this paper, a multi-scale bi-structural tensor with a certain anti-noise and robustness is introduced [7]. The structural tensor matrix is weighted by the spatial distance and gradient distance relationship, and the influence of noise is suppressed. Wherein the gradient distance $d_{i}^{g}$ and the spatial distance $d_{i}^{s}$ are defined as follows:

$$
\begin{aligned}
& d_{i}^{g}=\sqrt{\left(I_{x}\left(x_{i}, y_{i}\right)-I_{x}\left(x_{0}, y_{0}\right)\right)^{2}+\left(I_{y}\left(x_{i}, y_{i}\right)-I_{y}\left(x_{0}, y_{0}\right)\right)^{2}} \\
& d_{i}^{s}=\sqrt{\left(x_{i}-x_{0}\right)^{2}+\left(y_{i}-y_{0}\right)^{2}}
\end{aligned}
$$

In the formula (6) and (7), $d_{i}^{g}$ and $d_{i}^{s}$ represent the spatial distance and gradient distance at the point $\left(x_{i}, y_{j}\right)$ to the center point $\left(x_{0}, y_{0}\right)$ respectively. $\left(x_{i}, y_{j}\right)$ is a point in the local neighborhood $W$ centered on the point $\left(x_{0}, y_{0}\right)$. The weight functions $N(i)$ based on the gradient distance and the spatial distance are given as follows:

$$
N_{\rho, \sigma}(i)=\frac{1}{C_{\rho, \sigma}} \exp \left(-\frac{\left(d_{i}^{s}\right)^{2}}{2 \rho^{2}}\right) \cdot \exp \left(-\frac{\left(d_{i}^{g}\right)^{2}}{2 \sigma^{2}}\right)
$$

In the formula (8) $\rho$ and $\sigma$ denote the control gradient distance and the space distance parameter respectively. $C_{\rho, \sigma}$ is the normalization parameter, and the definition is as follows: 


$$
C_{\rho, \sigma}=\sum_{W} \exp \left(-\frac{\left(d_{i}^{s}\right)^{2}}{2 \rho^{2}}\right) \cdot \exp \left(-\frac{\left(d_{i}^{g}\right)^{2}}{2 \sigma^{2}}\right)
$$

The initial structure tensor $S_{0}$ in the formula (3) is rewritten using the formula (8) and rewrite the structural tensor with noise immunity as $S_{\rho, \sigma}(x, y)$.it is defined as follows:

$$
S_{\rho, \sigma}(x, y)=\left[\begin{array}{cc}
\sum_{\left(x_{i}, y_{j}\right)} N_{\rho, \sigma}(i)\left(I_{x}(x, y)\right)^{2} & \sum_{\left(x_{i}, y_{j}\right)} N_{\rho, \sigma}(i) I_{x}(x, y) I_{y}(x, y) \\
\sum_{\left(x_{i}, y_{j}\right)} N_{\rho, \sigma}(i) I_{y}(x, y) I_{x}(x, y) & \sum_{\left(x_{i}, y_{j}\right)} N_{\rho, \sigma}(i)\left(I_{y}(x, y)\right)^{2}
\end{array}\right]
$$

\section{Weight function rewrite}

The eigenvalues can be used to describe the inter texture information. According to the principle of similarity between similar blocks in non-local mean filtering, the eigenvalues are used to describe the local texture information of the image, but can not describe the similarity between the whole structural blocks. In [14], the matching similarity between image blocks is described by the similarity between matrices. At this time, the texture structure of the image can be represented by a matrix of structural tensors. Therefore, the texture of the image can be expressed in the form of a matrix of structural tensors. According to the similarity measure in [15] The similarity between $S_{\omega}(i)$ and $S_{\omega}(j)$ is descibed as $d\left(S_{\omega}(i), S_{\omega}(j)\right)$, defined as follows:

$$
d\left(S_{\omega}(i), S_{\omega}(j)\right)=\sqrt{\operatorname{tr}\left(\log \left(S_{\omega}(i)\right)-\log \left(S_{\omega}(i)\right)\right)^{2}}
$$

In the NLM-BST algorithm, the noise image $v=\{v(i) \mid i \in I\}$ is defined, the denoised image is $N L_{n n}[v](j)$. The pixel value at any point is calculated by the weighted average of all the pixel values in the image. The filtering formula is as follows:

$$
N L_{n n}[v](i)=\sum_{j \in I} w_{n n}(i, j) \cdot v(j)
$$

In formula (10), $I$ represents the neighborhood of pixel $j$ as the center, and the weight function ${ }_{0} \mathrm{f} w(i, j)$ in formula (2) is modified by formula (11). The improved weight function $w_{n n}(i, j)$ is defined as follows:

$$
w_{n n}(i, j)=\frac{1}{Z(i)} \exp \left(-\frac{\|v(N)-v(N)\|_{2, a}^{2}}{h^{2}}+\alpha \frac{\left(d\left(S_{w}(i)-S_{w}(j)\right)\right)^{2}}{h^{2}}\right)
$$

In the formula (13), $\alpha$ is a balance factor between $\left\|v\left(N_{i}\right)-v\left(N_{j}\right)\right\|_{2, a}^{2}$ and $\left|d\left(S_{\omega}(i)-S_{\omega}(j)\right)\right|^{2}$ and $h$ is the attenuation factor .

\section{Algorithm complexity analysis}

Suppose the size of the image is $W \times H$, the size of the filter window is $S \times S$, and the size of the search area is $F \times F$. The time complexity of the traditional NLM algorithm is mainly embodied in the similar block weight calculation. The time complexity of NLM algorithm is $O\left(S^{2} W H F^{2}\right)$.

The time complexity of the NLM-BST algorithm is based on the NLM algorithm, which increases the constraint of the structural tensor to the similarity computation and the tensor part of the NLM-BST algorithm is $T \times T$, the texture part of the tensor part of the NLM-BST algorithm Some of 
the time complexity is $O\left(\left(\frac{1}{2} F+T^{2}\right) W H\right)$.

\section{Analysis of results}

Experiments were carried out with gray scale images of Bridge, Chair and Barbara. The image resolution was $512 \times 512$,Bit depth of 8 , and the standard deviation of the image were added $15,20,25,30,35,40$ Gaussian white noise. To illustrate the NLM-BST.The validity of the algorithm is compared with that of traditional NLM. The subjective denoising effect map is denoised at a standard deviation of 30.Processing, image a, c, e, respectively, and g, i, k contrast analysis, NLM-BST algorithm after processing the overall image clarity is higher;The details of denoising for different images are compared and analyzed by images b, d, f and h, j, l, and the details of NLM-BST.The degree of visualization is high and the texture is clear. To further illustrate the visual sensory reliability, combined with the denoising data in Table 1 analysis.

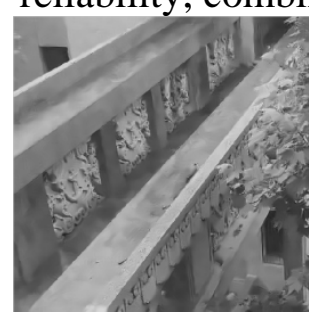

(a)

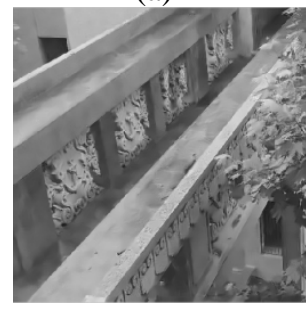

(g)

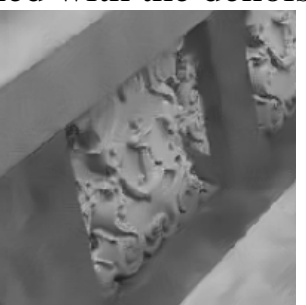

(b)

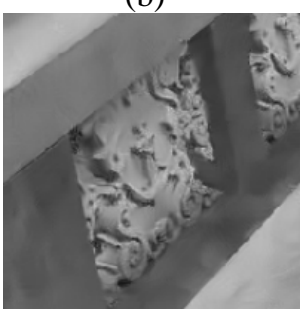

(h)

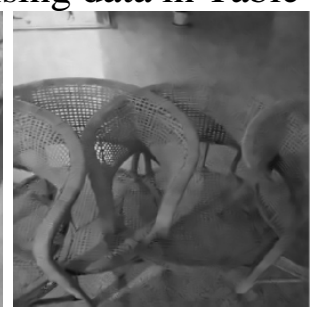

(c)

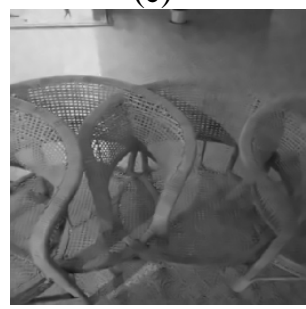

(i)

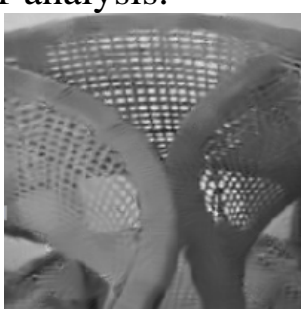

(d)

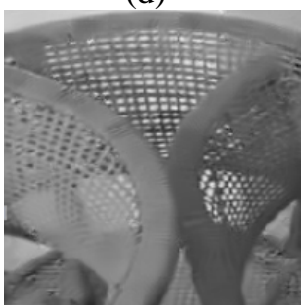

(j)

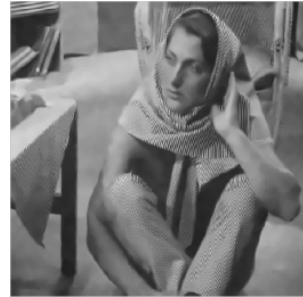

(e)

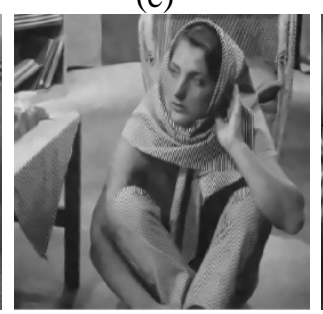

(k)

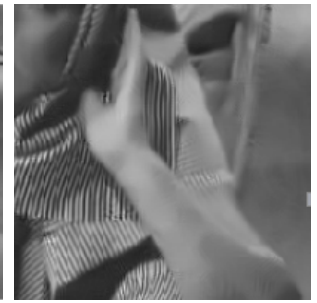

(f)

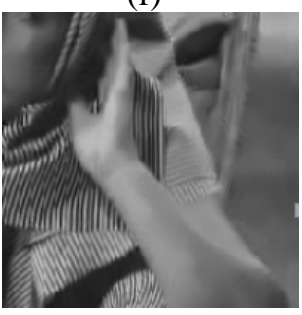

(l)

Figure 2. NLM and NLM-BST overall and local image denoising effect

Table 1 Peak SNR (dB) values for conventional NLM and NLM-BST denoising

\begin{tabular}{|c|c|c|c|c|c|c|c|}
\hline \multirow{2}{*}{ Image } & \multirow{2}{*}{ Method } & \multicolumn{6}{|c|}{ Noise $(\sigma)$} \\
\hline & & 15 & 20 & 25 & 30 & 35 & 40 \\
\hline \multirow{2}{*}{ Bridge } & $N L M$ & 28.721 & 27.241 & 26.137 & 25.246 & 24.489 & 23.905 \\
\hline & NLM-BST & 29.163 & 27.780 & 26.736 & 25.819 & 25.018 & 24.112 \\
\hline \multirow{2}{*}{ Chair } & $N L M$ & 30.583 & 29.117 & 28.090 & 27.301 & 26.700 & 26.227 \\
\hline & NLM-BST & 31.017 & 29.664 & 28.631 & 27.889 & 27.146 & 27.205 \\
\hline \multirow{2}{*}{ Barbara } & $N L M$ & 32.710 & 31.181 & 29.976 & 29.092 & 28.305 & 27.486 \\
\hline & NLM-BST & 33.003 & 31.661 & 30.554 & 29.601 & 29.014 & 28.337 \\
\hline
\end{tabular}

\section{Summary}

In this paper, a tensor model with some anti-noise and texture-preserving properties is constructed by using the gradient distance and spatial distance of the bilateral structural tensors, and the existing models are used to rewrite the NLM for the noise and gradient sensitivity. Weight function to improve the accuracy of similar block matching. Compared with traditional NLM algorithm,not only achieves good peak signal-to-noise ratio, but also improves sensory quality. This paper does not address how to maintain the similarity of the structural tensor in the similar rotation and how to find a better similarity matching metric which needs further study.

\section{References}


[1] Buades A, Coll B, Morel J M. A Non-Local Algorithm for Image Denoising[C]// IEEE Computer Society Conference on Computer Vision \& Pattern Recognition. 2005:60-65 vol.2.

[2] LIANG Guang-shun, WANG Ri-wei, WEN Xian-bin.Image Denoising Based on Bilateral Filter and Non-local Mean, J. Optoelectronics · Laser. (2015) 2231-2235.

[3] YUAN Yuan-qiang, LU Dong-ming, YUAN Yuan.An Improved Non-local Means Image De-noising Algorithm Using Mahalanobis Distance, J. Journal of Computer Aided Design \& Computer Graphics. 28(3) (2016) 404-410.

[4] Tasdizen T. Principal neighborhood dictionaries for nonlocal means image denoising[J]. Image Processing, IEEE Transactions on. 18(12) (2009) 2649-2660.

[5] Deledalle C A, Duval V, Salmon J. Non-local methods with shape-adaptive patches (NLM-SAP)[J]. Journal of Mathematical Imaging and Vision. 43(2) (2012) 103-120.

[6] Yan R, Shao L, Cvetkovic S D, et al. Improved nonlocal means based on pre-classification and invariant block matching, J. Display Technology, Journal of. 8(4) (2012) 212-218.

[7] Zhang L, Zhang L, Zhang D. A Multi-Scale Bilateral Structure Tensor Based Corner Detector[M]//Computer Vision-ACCV 2009. Springer Berlin Heidelberg. (2009) 618-627.

[8] Förstner W, Gülch E. A fast operator for detection and precise location of distinct points, corners and centres of circular features[C]/Proc. ISPRS intercommission conference on fast processing of photogrammetric data. (1987) 281-305.

[9] Wen Keli and Pian Zhaoyu, "The application of structure tensor in ddge detection," 2010 International Conference on Computer Application and System Modeling (ICCASM 2010), Taiyuan, 2010, pp. V4-274-V4-277.

[10]Zhao-Yu P, Gao L Q, Li G. An Image Segmentation Algorithm Based on Structure Tensor and Random Walk, J. Dongbei Daxue Xuebao/journal of Northeastern University, 30(30) (2009) 1095-1098.

[11]Caixia Li, Chanjuan Liu, Yilei Wang. Texture Image Denoising Algorithm Based on structure Tensor and Total Variation[C] Proceeding INCOS '13 Proceedings of the 2013 5th International Conference on Intelligent Networking and Collaborative Systems Pages 685-690.

[12]Weickert J. Scale-space properties of nonlinear diffusion filtering with a diffusion tensor[M]. Universität Kaiserslautern. Fachbereich Mathematik. Arbeitsgruppe Technomathematik, 1994.

[13]F. Catte, P. L. Lions, J. Morel, et al. Image selective smoothing and edge detection by nonlinear diffusion, J. SIAM J. Numer Anal, 29(1) (1992) 182-193.

[14]LI Dong-hai, LI Tong-liang, DUAN Wei-xia, et al.Optimal block matching algorithm based on matrix similarity and its application in image restoration, J . 41(1) (2014) 307-310.

[15]ZHANG Rui, FENG Xiang-Chu, WANG Si-Qi, et al.Non-local Image Denoising Algorithm Based on Sparse Gradient Field, J .Acta Automatica Sinica, 41(9) (2015) 1542-1552.

[16]Kang B, Choi O, Kim J D, et al. Noise reduction in magnetic resonance images using adaptive non-local means filterin, J. Electronics Letters. 49(5) (2013) 324-326.

[17]WANG Zhi-ming, ZHANG Li.Adaptive fast non-local image denoising algorithm, J .Journal of Image and Graphics. 14 (4) (2009) 669-675. 